\title{
Identifying Patterns of Lifestyle Behaviors among People with Type 2 Diabetes in Tianjin, China: A Latent Class Analysis
}

\author{
Xuying Wang · Jiageng Chen · Xiaoqian Liu · Fei Gao • \\ Haozuo Zhao · Duolan Han · Xiyue Jing • Yuanyuan Liu • \\ Zhuang Cui $\cdot$ Changping Li $\cdot$ Jun Ma
}

Received: August 22, 2017 / Published online: November 1, 2017

(C) The Author(s) 2017. This article is an open access publication

\section{ABSTRACT}

Introduction: Lifestyle behaviors are essential elements of diabetes care. The aims of this study were to identify distinct subgroups of people with type 2 diabetes based on personal levels of lifestyle behaviors and explore the different characteristics across these subgroups.

Methods: In 2015 and 2016, 1504 outpatients with a diagnosis of type 2 diabetes were selected via two-stage simple random sampling from 10 municipal district hospitals in Tianjin. Participants accepted an invitation by experienced

Xuying Wang, Jiageng Chen and Xiaoqian Liu contributed equally to this work.

Enhanced content To view enhanced content for this article go to http://www.medengine.com/Redeem/ 46CCF060741CB34D.

Electronic supplementary material The online version of this article (doi:10.1007/s13300-017-0327-7) contains supplementary material, which is available to authorized users.

X. Wang $\cdot$ J. Chen $\cdot$ X. Liu $\cdot$ F. Gao $\cdot$ H. Zhao

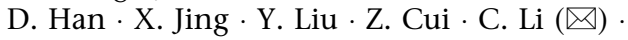

J. Ma $(\bowtie)$

Department of Health Statistics, College of Public

Health, Tianjin Medical University, Heping District,

Tianjin, People's Republic of China

e-mail: lichangping@tmu.edu.cn

J. Ma

e-mail: junma@tmu.edu.cn physicians to complete a questionnaire containing demographic and lifestyle content. Clinical data were collected by reviewing medical records. Latent class analysis was applied to identify patterns of lifestyle behaviors. Multinomial logistic regression was used to investigate the characteristics of the subgroups.

Results: The final model yielded a four-class solution: the healthy behavioral group, unhealthy diet and less activity group, smoking and drinking group, and sedentary and extremely inactive group. Further analysis found that variables, including age, sex, general/central obesity, treatment modalities, glycemic control, diabetes duration, and diabetes-related complications and comorbidities, were disproportionately distributed across the four latent classes $(P<0.05)$. Participants in the unhealthy diet and less activity group were more likely to have a longer duration of diabetes, poor glycemic control and more diabetes-related diseases relative to the other three latent classes.

Conclusions: Identification and characterization of subgroups based on lifestyle behaviors in individuals with type 2 diabetes can help health care providers to shift to targeted intervention strategies.

Keywords: Latent class analysis; Lifestyle behaviors; Type 2 diabetes mellitus 


\section{INTRODUCTION}

There has been a worldwide escalation in the prevalence of diabetes mellitus (DM) in the past decades [1]. The number of individuals living with DM is projected to reach 592 million by 2035 [2]. In China, the most populous country, the estimated prevalence of diabetes has increased dramatically from $1.3 \%$ in 1986 to $11.6 \%$ in 2010, translating into 113.9 million Chinese adults with DM [3,4] compared with the USA at 9.9\% [5] and Europe at 8.1\% [6]. Among the developing countries, China also has the largest population with diabetes [7]. Thus, DM has become one of the most alarming public health issues among all nations, especially China.

It is well established that unhealthy lifestyle choices may worsen the condition of type 2 diabetes. Insufficient physical activity, low consumption of vegetables, high salt intake and smoking are associated with poor glycemic control, exacerbating the development of both macro- and microvascular complications [8-11], and heavy drinking is associated with higher risks of cardiovascular events and all-cause mortality [12].

The evidence also shows that unhealthy behaviors often coexist within an individual [13-17]. For example, a German study reported that $66 \%$ of drinkers had at least one other unhealthy behavior, and $75 \%$ of smokers reported coexisting physical inactivity and/or an unhealthy diet [16]. Another study in England found that over $70 \%$ of adults who participate in one of the unhealthy behaviors also engaged in the others [17]. Additionally, compared with the simple sum of the separate effects, the combination of unhealthy behaviors is usually linked to a higher increased risk of mortality [17]. Therefore, it is essential to know whether we can identify subgroups with similar behaviors in individuals with type 2 diabetes so that interventions can be better tailored and targeted.

Latent class analysis (LCA), a flexible person-centered approach, has conceptual parallels to cluster analysis but is based on a measurement model much like factor analysis [18].
Individual variations inherently exist in the population but may not be clearly observed. LCA can distinguish the heterogeneity among individuals based on the response to observed variables (e.g., the presence or absence of specific behaviors) [18] and is widely used to explore patterns of multiple behaviors in different populations, such as children and adolescents, overweight/obese adults and older adults [13-15]. These study results have revealed that LCA is an innovative and effective method for grouping individuals with similar behaviors.

Evidence from diabetic studies, however, indicates that lifestyle behaviors are often separately studied [19-22]. Little is known about how these behaviors co-vary in populations with type 2 diabetes. Accordingly, the current study aimed to (1) explore the patterns of modifiable lifestyle behaviors (physical activity, sedentariness, diet, smoking and drinking) using LCA and (2) examine the demographic and clinical characteristic variations among distinct patterns, thereby identifying the subgroups of patients who are the most at risk. A full understanding of how the lifestyle behaviors cluster and whether typical risk subgroups can be identified on that basis could provide valuable and useful information to plan intervention strategies for individuals with type 2 diabetes.

\section{METHODS}

\section{Study Design and Patient Selection}

In 2015 and 2016, a cross-sectional study was conducted in Tianjin with two-stage simple random sampling. We included ten municipal districts consisting of six urban districts and four suburban districts. In the first stage, one healthcare insurance-assigned hospital was randomly selected from each district. At the next stage, patients who sought care between 23 November 2015 and 13 January 2016 were randomly selected. The inclusion criteria were as follows: being an outpatient with type 2 diabetes and covered by special disease outpatient service insurance (SDOSI); aged 18 years or older; being a local resident for at least 5 years 
consecutively before participation in our study; without mental illness, able to communicate verbally and able to provide informed consent. The exclusion criteria were as follows: having type 1 diabetes; having diabetes secondary to other diseases; pregnancy or breastfeeding. Our research was neither experimental nor interventional. Therefore, a trial register was not conducted.

\section{Data Collection}

Individuals were informed about the objective of the study and were asked to sign a consent form before the initiation of data collection. In the current study, we enrolled patients who were covered by SDOSI because each had a unique personal code comprising ten digits to represent themselves in the Municipal Human Resources and Social Security Bureau System. Participants accepted an invitation by experienced physicians to complete a questionnaire. They only needed to fill in their own personal code, which was recorded on their Medicare Card. Personal information such as name and phone number were not collected. Therefore, the anonymity of the participants' personal information was preserved.

In total, 1585 questionnaires were collected. The response rate was $93.2 \%$, and 81 questionnaires were excluded because of missing data. Thus, the final sample size for this study was 1504

All procedures followed were in accordance with the ethical standards of the responsible committee on human experimentation (institutional and national) and with the Helsinki Declaration of 1964, as revised in 2013. Informed consent was obtained from all patients for being included in the study.

\section{Study Variables}

Demographic variables included age, sex, height, bodyweight and waist circumference. Height and bodyweight were self-reported and were used to calculate the body mass index (BMI) $\left(\mathrm{kg} / \mathrm{m}^{2}\right)$. Overweight and obesity were defined at cutoff points of 24 and $28 \mathrm{~kg} / \mathrm{m}^{2}$, respectively, according to the Working Group for Obesity in China [23]. Abdominal obesity was defined as a waist circumference larger than $90 \mathrm{~cm}$ in males and $85 \mathrm{~cm}$ in females, a more reasonable cutoff point, especially for Chinese patients [24].

Clinical variables included a family history of diabetes, diabetes duration, HbA1c level, treatment modality and diabetes-related disease. A family history of diabetes was defined as at least one first-degree relative (parents, siblings or children) with type 2 diabetes. Diabetes duration was defined as the time from diagnosis to study entry. Treatment modality was classified into three groups: the oral antidiabetic agent-alone group (e.g., metformin and acarbose), the insulin-alone group (e.g., insulin aspart and insulin glargine) and the oral antidiabetic agent combined with insulin group. The measured $\mathrm{HbA} 1 \mathrm{c}$ and previous diagnosis of diabetes-related disease were ascertained by reviewing the medical records from secondary or tertiary hospitals in which the patients had ever been admitted. The HbA1c level was used to evaluate the glycemic status, where $<7 \%(53 \mathrm{mmol} / \mathrm{mol})$ was defined as good glycemic control and $\geq 7 \%(53 \mathrm{mmol} / \mathrm{mol})$ was defined as poor glycemic control [25]. Diabetes-related disease included macrovascular complications (cardiovascular disease, cerebrovascular disease and peripheral vascular disease), microvascular complications (diabetic nephropathy, diabetic retinopathy and diabetic peripheral neuropathy) and diabetes-related comorbidities (hypertension, hyperlipidemia and metabolic syndrome). The detailed definitions of diabetes and its complications are described below.

\section{Definitions of Diabetes and Its Complications} Diabetes was defined based on characteristic symptoms of diabetes (thirst, polyuria, polyphagia and weight loss) plus either a fasting plasma glucose level $\geq 7.0 \mathrm{mmol} / \mathrm{l}(126 \mathrm{mg} / \mathrm{dl})$ or a 2 -h post glucose load $\geq 11.1 \mathrm{mmol} / \mathrm{l}$ $(200 \mathrm{mg} / \mathrm{dl})$ [26].

Cardiovascular diseases included ischemic heart disease with an abnormal electrocardiography or stress test, myocardial infarction with typical changes on electrocardiography and 
plasma enzyme testing, coronary revascularization, percutaneous transluminal coronary angioplasty or coronary atherectomy [27].

Cerebrovascular diseases included asymptomatic cerebral atherosclerosis, stroke (ischemic and hemorrhagic stroke), cerebral small-vessel disease and acute cerebral vascular disease [28].

Peripheral vascular disease (PVD) was defined as an ankle arm blood pressure ratio $<0.8$, either at rest or after exercise, or a history of amputation due to PVD [27].

Diabetic nephropathy was defined as having persistent proteinuria, i.e., urinary albumin excretion rate (UAER) $\geq 20 \mu \mathrm{g} / \mathrm{min}$ or urinary albumin (UA) $\geq 30 \mathrm{mg} / 24 \mathrm{~h}$, after excluding other causes of kidney damage, urinary system infection and blood in the urine [29].

The definition of diabetic retinopathy was based on typical changes of retinopathy on funduscopic examination due to diabetes, including background, pre-proliferative and proliferative retinopathy or maculopathy [29].

Diabetic peripheral neuropathy was diagnosed if abnormalities were found in two or more of the described criteria: (1) the presence of one or more symptoms; (2) the absence of two or more ankle or knee reflexes; (3) abnormal vibration perception threshold related to age-related measures; (4) abnormal autonomic function as assessed by postural hypotension with a fall in the systolic blood pressure of $30 \mathrm{mmHg}$ or more and/or loss of heart rate variability $(\mathrm{R}-\mathrm{R}$ ratio $<1)$ [30].

\section{Latent Class Indicators}

The measures of latent class indicators included nine items that represent multiple dimensions of lifestyle behaviors. Binary latent class indicators were created to best reflect existing health recommendations and/or national guidelines, as described in detail below (Table S1).

\section{Physical Activity}

Participants reported the frequency and duration per week that they spent engaged in vigorous and moderate-intensity physical activity (i.e., activities that cause the body to heat up and sweat slightly, 50-70\% of the maximum heart rate). The two items were combined to determine whether patients met the recommendations of the Chinese Diabetes Society as well as American Diabetes Association (ADA): adults with diabetes should be encouraged to engage in at least $150 \mathrm{~min} /$ week of moderate-intensity aerobic physical activity, spread over at least 3 days/ week [24, 31]. Another item assessing the level of physical activity was whether the participants had engaged in regular exercise (allowing no more than 2 days to elapse between exercise sessions) during the past 3 months.

\section{Sedentary Behavior}

Participants were asked to report how many hours per day they usually spent on sedentary activities (i.e., working at home or the office; watching television; reading books or newspapers) and whether they engage in long-time sitting (defined as more than $90 \mathrm{~min}$ ). The ADA recommends that all individuals, including those with diabetes, should reduce sedentary time, particularly by breaking up long-time sitting $(90 \mathrm{~min})$ [31]. Considering the national provision of working time is $8 \mathrm{~h}$, we defined exceeding the recommended sedentary time as more than $90 \mathrm{~min}$ and the total time as $>8 \mathrm{~h}$ per day.

\section{Dietary Habits}

Healthy nutrition recommendations for the general public are also suitable for the population with type 2 diabetes [32]. Thus, in the present study, the consumption of staple foods, the main energy source, was dichotomized: $>400$ and $\leq 400$ g/day; cooking oil: $>30$ and $\leq 30$ g/day; salt: $>6$ and $\leq 6$ g/day, consistent with the recommendations of the Chinese Nutrition Society [33]. People who reported daily consumption exceeding these criteria were identified as exhibiting unhealthy eating behavior. Additionally, patients who reported a daily consumption of vegetables of at least $400 \mathrm{~g}$ were identified as exhibiting healthy eating behavior. 


\section{Smoking and Drinking}

The Chinese Diabetes Society suggests all patients avoid smoking and does not recommend drinking, especially for patients with type 2 diabetes [24]. Participants were asked whether they were current smokers or drinkers. A current smoker refers to smoking $>1$ cigarette/day and sustained $>1$ year; a current drinker refers to daily alcohol intake of $25 \mathrm{~g}$ or higher for males and $15 \mathrm{~g}$ for females, sustained $>1$ year. The two items were dichotomously coded.

\section{Statistical Analyses}

\section{Latent Class Analysis}

Latent class analysis (LCA) is an effective and flexible person-centered approach to group individuals with similar characteristics and is widely used to explore the observed patterns of multiple behaviors [13-15, 18, 21]. A series of latent class models beginning with a baseline one-class solution were conducted. The appropriate number of classes was chosen based on a combination of model-fit indexes and interpretability as follows [34, 35]: (1) $G^{2}$ statistic: generally, $G^{2}$ estimates that are less than the model's degrees of freedom signify a reasonably good model fit; (2) the Akaike information criterion (AIC), Bayesian information criterion (BIC) and sample-size-adjusted Bayesian information criterion (ssABIC): for all of these information criteria, a lower value indicates an improved model fit and a more optimal balance between the model fit and parsimony; (3) average latent class probabilities for most likely membership (ACPs), where ACPs approaching or exceeding 0.80 were desired; (4) model interpretability (e.g., each class should be distinguishable from the others based on the item-response probabilities, no class should be trivial in size, i.e., with a near-zero probability of membership); it should be possible to assign a meaningful label to each class.

\section{Descriptive and Univariate Analysis}

After model selection, individuals were assigned to the class in which they had the highest posterior probability of membership. Characteristic differences across latent classes were examined.
The data were given as medians (interquartile range) for continuous variables and proportions for categorical variables. After checking the distribution of continuous variables using a normality test, we used the Kruskal-Wallis test for non-normal distributions to compare continuous variables across groups. Categorical variables were compared using a chi-square test or the Kruskal-Wallis test for ordinal variables.

\section{Multinomial Logistic Regression}

To facilitate specific target group intervention measures, indicators of the subgroups were investigated using multinomial logistic regression, with class 1 as the reference category. Variables that are included in multivariate analysis were selected based on the results of univariate analysis. Age was divided into three groups according to the definition of the World Health Organization: youth ( $<45$ years), middle aged (45-64 years) and elderly ( $\geq 65$ years) [36]. The numbers of diabetes-related comorbidities, macro- and microvascular complications per patient were calculated.

LCA analyses were performed using SAS PROC LCA (version 1.3.2) [34], and the remaining analyses were conducted using SAS 9.3 (SAS Institute, Cary, NC, USA). $P<0.05$ (two-sided) was considered statistically significant.

\section{RESULTS}

\section{Participant Characteristics}

The study included 1504 patients (741 males and 763 females) with type 2 diabetes, and the median age was 63 years $(\mathrm{IQR}=13)$. The characteristics of all participants are shown in Tables 2 and 3. Over half of the sample had abdominal obesity (59.4\%), while nearly half had a family history of diabetes $(47.1 \%)$. The median HbA1c level was 7.5\%, and only $23.5 \%$ of patients reached the HbA1c target according to the ADA criteria (HbA1c level less than 7.0\%) [25]. The median duration of type 2 diabetes was 9 years, and $69.7 \%$ of patients had diabetes for at least 5 years. 


\section{Latent Class Analysis}

\section{Number of Latent Classes}

Models with one through five latent classes were estimated (Table 1 ). The $G^{2}$ estimates, as well as the three information criteria indices (AIC, BIC and ssABIC), decreased substantially for models with 1-4 classes and moderately for additional classes. BIC was suggested to be the best indicator to decide the number of latent classes in mixture modeling among the traditionally used information criteria [37]. Here, the four-class model had the lowest BIC value. The ACPs, an indicator of the reliability of the LCA class assignments, was highest for models with four classes. Considering conceptual implications, the final model yielded a four-class solution.

\section{Latent Class Profiles}

Each latent class corresponds to an underlying subgroup of participants characterized by a special behavioral pattern. The probability of reporting each of the nine behaviors within the generated classes was used to characterize the different profiles of individuals (Fig. 1). Class 1 represented more than half of the participants with a healthy pattern relative to the other three classes and was labeled the "healthy behavioral group." Individuals in this class had relatively high probabilities of participating in physical activity, being moderately sedentary, following a daily healthy diet and having low probabilities of being a current smoker or drinker. Class 2 accounted for $4.0 \%$ of the sample and can be characterized by their high probability of reporting unhealthy behaviors, such as low participation in physical activity, slightly higher sedentary time, low vegetable consumption, high intake of staple food, cooking oil and salt, but low probabilities of being a current smoker or drinker. As such, class 2 was labeled the "unhealthy diet and less activity group." Class 3 represented $8.3 \%$ of the sample and was distinguished from other classes by the highest probability of being a current smoker and drinker and was labeled the "smoking and drinking group." Class 4 accounted for the second largest segment of the sample with an estimated prevalence of $28.5 \%$. Individuals in this class had a pattern of the lowest probability of engaging in regular exercise or adequate physical activity and tended to have relatively high probabilities of having sedentary behaviors. Thus, we labeled this class the "sedentary and extremely inactive group."

\section{Characteristics of Latent Class Membership}

\section{Demographic Factors}

All participants were assigned to the class in which they had the highest posterior probability of membership. The results were presented in the form of column percentages, which showed that these classes varied by demographic characteristics (Table 2). Class 3 had the highest proportion of members who were $45-64$ years of age $(P=0.001)$. Class 4 comprised mostly individuals older than 65 years

Table 1 Model fit statistics of the one- to five-class LCA models $(n=1504)$

\begin{tabular}{lllllrl}
\hline Number of classes & $\boldsymbol{G}^{\mathbf{2}}$ & $\boldsymbol{D F}$ & \multicolumn{1}{l}{ AIC } & BIC & ssABIC & ACPs \\
\hline 1 & 1194.22 & 502 & 1212.22 & 1260.06 & 1231.47 & - \\
2 & 816.70 & 492 & 854.70 & 955.70 & 895.34 & 0.89 \\
3 & 584.64 & 482 & 642.64 & 796.80 & 704.68 & 0.88 \\
4 & $\mathbf{3 9 5 . 5 6}$ & $\mathbf{4 7 2}$ & $\mathbf{4 7 3 . 5 6}$ & $\mathbf{6 7 0 . 5 7}$ & $\mathbf{5 1 4 . 9 1}$ & $\mathbf{0 . 9 0}$ \\
5 & 312.09 & 462 & 410.09 & 680.87 & 556.98 & 0.86 \\
\hline
\end{tabular}

Bold font signifies the selected model

$G^{2}$ Likelihood ratio chi-square, $D F$ degrees of freedom, $A I C$ Akaike's Information Criterion, BIC Bayesian Information Criterion, ss $A B I C$ sample-size adjusted BIC, $A C P s$ average latent class probabilities for most likely latent class membership 


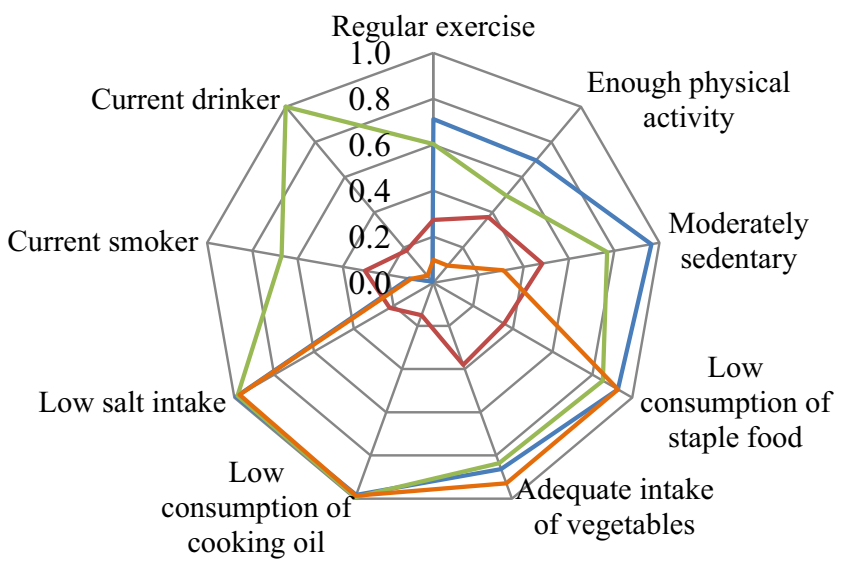

- Healthy behavioral group $(59.2 \%)$

unhealthy diet and less activity group $(4.0 \%)$

Smoking and drinking group $(8.3 \%)$

- sedentary and extremely inactivity group $(28.5 \%)$

Fig. 1 Item-response probabilities of lifestyle behaviors for the four-class model: probability of endorsing an item given a latent class. Item-response probabilities are the probabilities of participants responding to different items

relative to the remaining latent classes $(P=0.002)$. Within each subgroup, class 3 was composed predominantly of males, and class 4 participants comprised mostly females $(P<0.001)$. Normal and overweight defined by BMI were similar between classes $(P=0.574$ and $P=0.176$, respectively). However, a higher proportion of individuals in class 2 and class 4 were classified as obese compared with those in class 1 and class $3(P=0.003)$. Specifically, abdominal obesity was much more frequently observed in class 2 relative to the remaining latent classes $(P=0.037)$.

\section{Clinical Factors}

The clinical factors were disproportionately distributed across four latent classes (Table 3). Compared with class 1 , participants in class 2 accounted for a higher proportion treated with oral antidiabetic drugs combined with insulin $(63.3 \%$ vs. $51.5 \%)$, had a longer duration of diabetes (duration $\geq 10$ years: $55.0 \%$ vs. $34.3 \%$ ) and developed more diabetes-related complications and comorbidities (such as more than one macrovascular complication: $30.0 \%$ vs. $15.3 \%$; more than one microvascular complication: $30.0 \%$ vs. $18.9 \%$; more than one comorbidity: $43.3 \%$ vs. $21.9 \%$ ). The percentages of shorter diabetes duration (duration $<5$ years: $41.0 \%$ vs. $15.0 \%)$ as well as glycemic control achieved depending on latent class membership. For example, among those in the healthy behavioral group, the probability of endorsing regular exercise was 0.7

(30.4\% vs. $18.3 \%)$ were highest in class 3 . People in class 2 and class 4 had a lower proportion at the glycemic control goal (18.3\% and $19.3 \%$, respectively). Family history had a higher proportion in class $2(60.0 \%$ vs. $45.5 \%)$, although not significantly $(P=0.215)$.

\section{Multivariable Multinomial Logistic Model}

Table 4 shows the results of multinomial logistic regression analysis: people who were obese and had poor glycemic control had significantly higher odds of being in classes 2 and 4 than in class 1 . Members who had abdominal obesity, a longer duration of diabetes and more diabetes-related complications and comorbidities seemed to have significantly higher odds of being in class 2 . Patients of male gender and age 45 to 64 years had significantly higher odds of being in class 3 . However, female gender had significantly higher odds of being in class 4 .

\section{DISCUSSION}

Our study applied LCA to capture meaningful patterns of lifestyle behaviors in individuals with type 2 diabetes. Four distinct groups were revealed in the current study, in which characteristic disparities were obvious. It may be 
Table 2 Demographic characteristics of individuals with type 2 diabetes according to latent class

\begin{tabular}{|c|c|c|c|c|c|c|}
\hline Characteristics & Overall & Class 1 & Class 2 & Class 3 & Class 4 & $P$ value \\
\hline N (\%) & $1504(100)$ & $890(59.2)$ & $60(4.0)$ & $125(8.3)$ & $429(28.5)$ & \\
\hline Age (years) & $63(13)$ & $63(12)$ & $63(10)$ & $60(13)$ & $64(13)$ & 0.019 \\
\hline \multicolumn{7}{|l|}{ Age groups (years) } \\
\hline$<45$ & $70(4.6)$ & $38(4.3)$ & $2(3.3)$ & $6(4.8)$ & $24(5.6)$ & 0.707 \\
\hline $45-64$ & $787(52.3)$ & $477(53.6)$ & $35(58.3)$ & $83(66.4)$ & $192(44.8)$ & 0.001 \\
\hline$\geq 65$ & $647(43.0)$ & $375(42.1)$ & $23(38.3)$ & $36(28.8)$ & $213(49.7)$ & 0.002 \\
\hline Sex (males) & $741(49.3)$ & $436(49.0)$ & $33(55.0)$ & $109(87.2)$ & $163(38.0)$ & $<0.001$ \\
\hline \multicolumn{7}{|l|}{ BMI groups } \\
\hline Normal & $432(29.0)$ & $264(30.0)$ & $14(23.3)$ & $32(26.0)$ & $122(28.4)$ & 0.574 \\
\hline Overweight & $713(47.8)$ & $438(49.8)$ & $29(48.3)$ & $62(50.4)$ & $184(42.9)$ & 0.176 \\
\hline Obesity & $346(23.2)$ & $177(20.1)$ & $17(28.3)$ & $29(23.6)$ & $123(28.7)$ & 0.003 \\
\hline WC & $89(14)$ & $88.5(13.2)$ & $91.5(11.7)$ & $92.0(13.5)$ & $89.0(17.0)$ & 0.001 \\
\hline Abdominal obesity & $894(59.4)$ & $512(57.5)$ & $45(75.0)$ & $80(64.0)$ & $257(59.9)$ & 0.037 \\
\hline
\end{tabular}

Data were expressed as medians (IQR) or $n$ (\%)

$P$ values for the difference between subgroups were calculated with the Kruskal-Wallis test or chi-square test, as appropriate Bold values indicate major characteristics

$B M I$ body mass index, $W C$ waist circumference, Class 1 healthy behavioral group, Class 2 unhealthy diet and less activity group, Class 3 smoking and drinking group; Class 4 sedentary and extremely inactive group

potentially helpful in targeting high-risk subgroups and developing specific intervention strategies.

Class 1 , the healthy behavioral group, was the most prevalent category (59.2\%) in our sample. Although the target level of HbA1c was not generally achieved, the individuals in class 1 had been engaging in a healthy lifestyle. One explanation is that the patients in class 1 changed their lifestyle during the illness because individuals in this class had diabetes for an average of 9 years. Thus, more satisfactory improvements still need long-term persistence.

Abdominal obesity, poor glycemic control, long diabetes duration and the development of diabetes-related diseases were more prevalent in the unhealthy diet and less activity group (Class 2 ). These findings are consistent with those of previous studies, which indicated the relationship between unhealthy lifestyle and unsatisfactory glycemic control $[9,38]$. Moreover, diabetes duration was significantly associated with worse glycemic control, and they both were risk factors for developing macroand microvascular complications $[39,40]$. These results are in line with our data showing that the higher prevalence of diabetes-related disease among class 2 members is mostly explained by poor glycemic control resulting from an unhealthy lifestyle and prolonged duration of diabetes. Abdominal obesity in class 2 was more prevalent relative to the other classes. Kissebah et al. first reported that women with central obesity were more inclined to have glucose intolerance, hyperinsulinemia and hypertriglyceridemia [41]. Evidence from a recent study demonstrated an independent and significant association of central obesity with unsatisfactory glycemic control and early nephropathy; physical activity interaction with central obesity was also found to be associated 
Table 3 Clinical characteristics of individuals with type 2 diabetes according to latent class

\begin{tabular}{|c|c|c|c|c|c|c|}
\hline Characteristics & Overall & Class 1 & Class 2 & Class 3 & Class 4 & $P$ value \\
\hline Family history of diabetes & $709(47.1)$ & $419(47.1)$ & $36(60.0)$ & $59(47.2)$ & $195(45.5)$ & 0.215 \\
\hline Treatment modalities & & & & & & $<0.001$ \\
\hline Oral antidiabetic agents alone & $601(40.0)$ & $351(39.4)$ & $20(33.3)$ & $57(45.6)$ & $173(40.3)$ & \\
\hline Insulin alone & $134(8.9)$ & $81(9.1)$ & $2(3.3)$ & $14(11.2)$ & $37(8.6)$ & \\
\hline Oral antidiabetic agents and insulin & $769(51.1)$ & $458(51.5)$ & $38(63.3)$ & $54(43.2)$ & $219(51.0)$ & \\
\hline HbAlc (\%) & $7.5(1.2)$ & $7.4(1.1)$ & $7.9(1.6)$ & $7.4(1.2)$ & $7.8(1.9)$ & $<0.001$ \\
\hline Glycemic control & $353(23.5)$ & $221(24.8)$ & $11(18.3)$ & $38(30.4)$ & $83(19.3)$ & $<0.001$ \\
\hline Mean duration of type 2 diabetes (years) & $9(9)$ & $9(9)$ & $12(11)$ & $8(9)$ & $9(9)$ & 0.013 \\
\hline Duration of type 2 diabetes (years) & & & & & & 0.004 \\
\hline $0-4.9$ & $455(30.3)$ & $264(29.7)$ & $9(15.0)$ & $51(41.0)$ & $131(30.5)$ & \\
\hline $5-9.9$ & $534(35.5)$ & $321(36.1)$ & $18(30.0)$ & $40(32.0)$ & $155(36.1)$ & \\
\hline$\geq 10$ & $515(34.2)$ & $305(34.3)$ & $33(55.0)$ & $34(27.0)$ & $143(33.3)$ & \\
\hline Macrovascular complications & & & & & & 0.033 \\
\hline 0 & $841(55.9)$ & $507(57.0)$ & $30(50.0)$ & $77(61.6)$ & $227(52.9)$ & \\
\hline 1 & $419(27.9)$ & $247(27.8)$ & $12(20.0)$ & $34(27.2)$ & $126(29.4)$ & \\
\hline$>1$ & $244(16.2)$ & $136(15.3)$ & $18(30.0)$ & $14(11.2)$ & $76(17.7)$ & \\
\hline Microvascular complications & & & & & & 0.004 \\
\hline 0 & $849(56.4)$ & $517(58.1)$ & $24(40.0)$ & $82(65.6)$ & $226(52.7)$ & \\
\hline 1 & $376(25.0)$ & $205(23.0)$ & $18(30.0)$ & $29(23.2)$ & $124(28.9)$ & \\
\hline$>1$ & $279(18.6)$ & $168(18.9)$ & $18(30.0)$ & $14(11.2)$ & $79(18.4)$ & \\
\hline Diabetes-related comorbidities & & & & & & 0.002 \\
\hline 0 & $645(42.9)$ & $395(44.4)$ & $18(30.0)$ & $63(50.4)$ & $169(39.4)$ & \\
\hline 1 & $489(32.5)$ & $300(33.7)$ & $16(26.7)$ & $34(27.2)$ & $139(32.4)$ & \\
\hline$>1$ & $370(24.6)$ & $195(21.9)$ & $26(43.3)$ & $28(22.4)$ & $121(28.2)$ & \\
\hline
\end{tabular}

Data were expressed as medians (IQR) or $n(\%)$

$P$ values for the difference between subgroups were calculated using the Kruskal-Wallis test or chi-square test, as appropriate Bold values indicate major characteristics

HbAlc glycated hemoglobin; targets of glycemic control were defined as HbAlc less than $7 \%(53 \mathrm{mmol} / \mathrm{mol})$, Class 1 healthy behavioral group, Class 2 unhealthy diet and less activity group, Class 3 smoking and drinking group, Class 4 sedentary and extremely inactive group

with the aforementioned outcomes [42]. Moreover, the relationship between dietary patterns and central obesity has been observed in older Chinese individuals [43]. Thus, interventions in individuals with longer diabetes duration and more diabetes-related diseases could focus on diet and exercise. People might need to learn their dual role. Not only is weight management by dietary and regular exercise able to reduce 
Table 4 Results of the logistic regression model investigating factors associated with class membership

\begin{tabular}{|c|c|c|c|c|c|c|}
\hline \multirow[t]{2}{*}{ Characteristic } & \multicolumn{2}{|c|}{ Class 2 vs. class 1} & \multicolumn{2}{|c|}{ Class 3 vs. class 1} & \multicolumn{2}{|c|}{ Class 4 vs. class 1} \\
\hline & OR & $95 \% \mathrm{CI}$ & OR & $95 \% \mathrm{CI}$ & OR & $95 \% \mathrm{CI}$ \\
\hline \multicolumn{7}{|l|}{ Age group (years) } \\
\hline$<45$ & Reference & & & & & \\
\hline $45-64$ & 1.04 & $0.23-4.72$ & $1.23^{*}$ & $1.01-1.65$ & 0.64 & $0.35-1.16$ \\
\hline$\geq 65$ & 0.66 & $0.14-3.11$ & 0.88 & $0.28-2.79$ & 1.18 & $0.48-1.60$ \\
\hline \multicolumn{7}{|l|}{ Sex } \\
\hline Women & Reference & & & & & \\
\hline Males & 1.40 & $0.80-2.45$ & $7.91^{*}$ & $4.34-14.41$ & $0.80^{*}$ & $0.59-0.98$ \\
\hline \multicolumn{7}{|l|}{ BMI groups } \\
\hline Normal & Reference & & & & & \\
\hline Overweight & 1.09 & $0.53-2.23$ & 1.06 & $0.64-1.77$ & 0.83 & $0.62-1.12$ \\
\hline Obesity & $1.22^{*}$ & $1.07-1.81$ & 1.47 & $0.78-2.78$ & $1.30^{*}$ & $1.09-1.75$ \\
\hline Abdominal obesity & $1.61^{*}$ & $1.03-2.53$ & 1.31 & $0.83-2.06$ & 0.99 & $0.76-1.29$ \\
\hline \multicolumn{7}{|l|}{ Treatment modalities } \\
\hline Oral antidiabetic agents alone & Reference & & & & & \\
\hline Insulin alone & 0.14 & $0.02-1.09$ & 1.40 & $0.67-2.94$ & 0.93 & $0.58-1.50$ \\
\hline Oral antidiabetic agents combined with insulin & 0.68 & $0.38-1.24$ & 0.73 & $0.46-1.16$ & 1.01 & $0.77-1.32$ \\
\hline Poor glycemic control & $1.45^{*}$ & $1.07-2.25$ & 0.66 & $0.42-1.05$ & $1.52^{*}$ & $1.05-2.12$ \\
\hline Duration of type 2 diabetes (years) & $1.56^{*}$ & $1.22-1.95$ & 0.91 & $0.86-1.04$ & 0.99 & $0.97-1.01$ \\
\hline Macrovascular complications & $1.28^{*}$ & $1.04-1.44$ & 1.18 & $0.78-1.79$ & 1.00 & $0.80-1.27$ \\
\hline Microvascular complications & $1.40^{*}$ & $1.13-1.80$ & 0.78 & $0.57-1.06$ & 0.98 & $0.84-1.16$ \\
\hline Diabetes-related comorbidities & $1.58^{*}$ & $1.15-2.12$ & 0.88 & $0.69-1.13$ & 1.08 & $0.94-1.24$ \\
\hline
\end{tabular}

OR odds ratio, CI confidence interval, Class 1 healthy behavioral group, Class 2 unhealthy diet and less activity group; Class 3 smoking and drinking group, Class 4 sedentary and extremely inactive group ${ }^{*} p<0.05$

visceral adipose tissue, it is also the cornerstone of any successful treatment of diabetes.

In addition to this, men with smoking and drinking behaviors were largely distributed in class 3. Systematic review and meta-analysis indicated that cigarette smoking is associated with an increased risk of type 2 diabetes and microvascular complications such as diabetic peripheral neuropathy $[44,45]$. Xie et al. summarized recent evidence showing that insulin action and pancreatic cell function can be affected by nicotine, further facilitating the development of diabetes complications [46]. Thus, smoking cessation is crucial for glycemic control and slows the development of diabetes vascular complications [8]. Regarding drinking behavior, heavy drinkers were at an elevated risk of poor health, whereas moderate alcohol consumption had an inverse association with the risk of type 2 diabetes, but this may be limited to women and non-Asian populations $[22,47]$. A recent study conducted on Chinese 
men living with type 2 diabetes suggested that avoiding tobacco and alcohol could have a prominent impact on their health status [48]. Interestingly, relatively more satisfactory glycemic control and fewer complications and comorbidities existed in this group. Possible reasons were the shorter duration of diabetes and adherence to other health behaviors. Therefore, it is imperative to class 3 members to be informed about the benefits of early intervention that focus on quitting smoking and limiting alcohol consumption.

Distinct from the above three classes, more women were included in the sedentary and extremely inactive group (class 4). Notably, the percentage of general obesity derived from BMI in this class was the highest, in agreement with prior research supporting that obesity is more common in women [49]. One reason is that fluctuations in hormone concentrations due to reproductive factors predispose women to excess weight gain [50]. Another reason is the high-risk time for women to gain weight, known as menopause [50]. In addition, the lack of exercise and sedentary behavior both play a fundamental role in the process of weight gain $[13,19]$. However, gender roles influenced by genetic and social factors place some women in the position of family caregiver, leaving little time or energy for exercise [51]. The American College of Sports Medicine and the American Diabetes Association hold the joint statement that physical activity and regular exercise can assist with the management of blood glucose levels [52]. Thus, lifestyle modification aimed at increasing regular exercise and reducing sedentary time is the principal therapy for members in class 4 , especially women with a higher BMI index.

The application of LCA showed the merits of identifying the heterogeneity that exists in type 2 diabetes patients. Four classes emerged from the person-centered approach characterized by different behavior profiles, indicating special needs in those with diabetes. The strengths of the study also include the relatively large size of the sample and diversity of participants. The distribution of participants was wide and spanned the urban areas, suburbs and far-suburbs in Tianjin, and the response to our study was
93.2\%. We believe our sample was a representative population, and the results were applicable to other patients in Tianjin, China.

The limitations of this study include the self-reported behavior measures, which are subject to recall bias and report bias. Accordingly, several intervals were offered to help participants to confirm their responses. In addition, data for socioeconomic variables such as education level, marital status and working status were not collected. Future research could involve these variables, which may provide more information that differentiates members across classes. Finally, but most importantly, the design of our study is cross-sectional. Therefore, we can only provide a snapshot of the association between current lifestyle behaviors and other characteristics in diabetes patients. However, four typical behavior patterns were identified in our data, which uncover the special needs of lifestyle interventions. A prospective cohort study is needed to evaluate whether targeted strategies can assist to maximize the potential effect of health interventions, both in the short term and over the long term.

\section{CONCLUSION}

Four categories have been identified based on lifestyle behavior factors in a type 2 diabetes population. The demographic and clinical characteristics of each group were evident, which can be potentially used to locate high-risk groups. Individualized behavioral modification strategies should be applied to specific high-risk groups.

\section{ACKNOWLEDGEMENTS}

The study was funded by the National Natural Science Foundation of China (71373175), Tianjin Municipal Human Resources and Social Security Bureau (TMIR 201502) and the Ministry of Education of Humanities and Social Science project (17YJAZH048). These grants also fund the article processing charges associated with the publication of this article. All authors 
had full access to all of the data in this study and take complete responsibility for the integrity of the data and accuracy of the data analysis. All named authors meet the International Committee of Medical Journal Editors (ICMJE) criteria for authorship for this manuscript, take responsibility for the integrity of the work as a whole and have given final approval for the version to be published. The authors would like to express their gratitude to all participants for their commitment to this research effort.

Disclosures. Xuying Wang, Jiageng Chen, Xiaoqian Liu, Fei Gao, Haozuo Zhao, Duolan Han, Xiyue Jing, Yuanyuan Liu, Zhuang Cui, Changping $\mathrm{Li}$ and Jun Ma have nothing to disclose.

Compliance with Ethics Guidelines. All procedures followed were in accordance with the ethical standards of the responsible committee on human experimentation (institutional and national) and with the Helsinki Declaration of 1964, as revised in 2013. Informed consent was obtained from all patients for participation in the study.

Data Availability. The data sets generated and/or analyzed during the current study are available from the corresponding author on reasonable request.

Open Access. This article is distributed under the terms of the Creative Commons Attribution-NonCommercial 4.0 International License (http://creativecommons.org/licenses/ by-nc/4.0/), which permits any noncommercial use, distribution, and reproduction in any medium, provided you give appropriate credit to the original author(s) and the source, provide a link to the Creative Commons license, and indicate if changes were made.

\section{REFERENCES}

1. Collaboration NCDRF. Worldwide trends in diabetes since 1980: a pooled analysis of 751 population-based studies with 4.4 million participants. Lancet 2016;387(10027):1513-30.
2. Guariguata L, Whiting DR, Hambleton I, et al. Global estimates of diabetes prevalence for 2013 and projections for 2035. Diabetes Res Clin Pract. 2014;103(2):137-49.

3. Pan XR, Li GW, Hu YH, et al. Effects of diet and exercise in preventing NIDDM in people with impaired glucose tolerance. The Da Qing IGT and Diabetes Study. Diabetes Care. 1997;20(4):537-44.

4. Xu Y, Wang L, He J, et al. Prevalence and control of diabetes in Chinese adults. JAMA. 2013;310(9):948-59.

5. Selvin E, Parrinello CM, Sacks DB, Coresh J. Trends in prevalence and control of diabetes in the United States, 1988-1994 and 1999-2010. Ann Intern Med. 2014;160(8):517-25.

6. Ryden L, Grant PJ, Anker SD, et al. ESC Guidelines on diabetes, pre-diabetes, and cardiovascular diseases developed in collaboration with the EASD: the Task Force on diabetes, pre-diabetes, and cardiovascular diseases of the European Society of Cardiology (ESC) and developed in collaboration with the European Association for the Study of Diabetes (EASD). Eur Heart J. 2013;34(39):3035-87.

7. Yang W, Lu J, Weng J, et al. Prevalence of diabetes among men and women in China. N Engl J Med. 2010;362(25):1090-101.

8. Sliwinska-Mosson M, Milnerowicz H. The impact of smoking on the development of diabetes and its complications. Diab Vasc Dis Res. 2017;14(4):265-76.

9. Bralic LV, Bergman MB, Vrdoljak D. The association of lifestyle and stress with poor glycemic control in patients with diabetes mellitus type 2: a Croatian nationwide primary care cross-sectional study. Croat Med J. 2015;56(4):357-65.

10. Badedi M, Solan Y, Darraj H, et al. Factors associated with long-term control of type 2 diabetes mellitus. J Diabetes Res. 2016;2016:2109542.

11. Mohan V, Shah S, Saboo B. Current glycemic status and diabetes related complications among type 2 diabetes patients in India: data from the A1chieve study. J Assoc Phys India. 2013;61(1 Suppl):12-5.

12. Blomster JI, Zoungas S, Chalmers J, et al. The relationship between alcohol consumption and vascular complications and mortality in individuals with type 2 diabetes. Diabetes Care. 2014;37(5):1353-9.

13. Patnode CD, Lytle LA, Erickson DJ, et al. Physical activity and sedentary activity patterns among children and adolescents: a latent class analysis approach. J Phys Act Health. 2011;8(4):457-67. 
14. Fitzpatrick SL, Coughlin JW, Appel LJ, et al. Application of latent class analysis to identify behavioral patterns of response to behavioral lifestyle interventions in overweight and obese adults. Int J Behav Med. 2015;22(4):471-80.

15. Sodergren M, Wang WC, Salmon J, et al. Predicting healthy lifestyle patterns among retirement age older adults in the WELL study: a latent class analysis of sex differences. Maturitas. 2014;77(1):41-6.

16. Schneider S, Huy C, Schuessler M, Diehl K, Schwarz S. Optimising lifestyle interventions: identification of health behaviour patterns by cluster analysis in a German $50+$ survey. Eur J Public Health. $2009 ; 19(3): 271-7$.

17. Poortinga $\mathrm{W}$. The prevalence and clustering of four major lifestyle risk factors in an English dult population. Prev Med. 2007;44(2):124-8.

18. Lanza ST, Rhoades BL, Nix RL, Greenberg MT. Modeling the interplay of multilevel risk factors for future academic and behavior problems: a person-centered approach. Dev Psychopathol. 2010;22(2):313-35.

19. Jakicic JM, Gregg E, Knowler W, et al. Activity patterns of obese adults with type 2 diabetes in the look AHEAD study. Med Sci Sports Exerc. 2010;42(11):1995-2005.

20. Jeppesen C, Bjerregaard P, Jorgensen ME. Dietary patterns in Greenland and their relationship with type 2 diabetes mellitus and glucose intolerance. Public Health Nutr. 2014;17(2):462-70.

21. Gariepy G, Malla A, Wang J, et al. Types of smokers in a community sample of individuals with type 2 diabetes: a latent class analysis. Diabet Med. 2012;29(5):586-92.

22. Hodge AM, English DR, O'Dea K, Giles GG. Alcohol intake, consumption pattern and beverage type, and the risk of type 2 diabetes. Diabet Med. 2006;23(6):690-7.

23. Zhou B. Predictive values of body mass index and waist circumference to risk factors of related diseases in Chinese adult population. Zhonghua Liu Xing Bing Xue Za Zhi. 2002;23(1):5-10.

24. The Chinese Diabetes Society. Guideline for prevention and treatment of type 2 diabetes in Chinese (2013 edition). Chin J Diabetes Mellitus. 2014;6(7):447-98.

25. American Diabetes Association. Glycemic targets. Sec. 6. In standards of medical care in Diabetesd-2017. Diabetes Care. 2017;40(Suppl. 1):S48-56.
26. World Health Orgnization. Definition and diagnosis of diabetes mellitus and intermediate hyperglycemia: report of a WHO/IDF consultation. Geneva: WHO Document Production Services; 2006.

27. Orchard TJ, Dorman JS, Maser RE, et al. Prevalence of complications in IDDM by sex and duration. Pittsburgh Epidemiology of Diabetes Complications Study II. Diabetes. 1990;39(9):1116-24.

28. Zhou H, Zhang X, Lu J. Progress on diabetic cerebrovascular diseases. Bosn J Basic Med Sci. 2014;14(4):185-90.

29. Li L, Ji L, Guo X, et al. Prevalence of microvascular diseases among tertiary care Chinese with early versus late onset of type 2 diabetes. J Diabetes Compl. 2015;29(1):32-7.

30. Tesfaye S, Stevens LK, Stephenson JM, et al. Prevalence of diabetic peripheral neuropathy and its relation to glycaemic control and potential risk factors: the EURODIAB IDDM complications study. Diabetologia. 1996;39(11):1377-84.

31. American Diabetes Association. Comprehensive medical evaluation and assessment of comorbidities. Sec. 3. In standards of medical care in Diabetesd-2017. Diabetes Care. 2017;40(Suppl. 1):S25-32.

32. Bantle JP, Wylie-Rosett J, Albright AL, et al. Nutrition recommendations and interventions for diabetes: a position statement of the American Diabetes Association. Diabetes Care. 2008;31(Suppl. 1):S61-78.

33. The China Nutrition Society. Chinese residents dietary guidelines-core recommendation. Chin J Woman Child Health Res. 2016;27(5):670.

34. Lanza ST, Collins LM, Lemmon DR, Schafer JL. PROC LCA: a SAS procedure for latent class analysis. Struct Equ Model. 2007;14(4):671-94.

35. Lanza ST, Rhoades BL. Latent class analysis: an alternative perspective on subgroup analysis in prevention and treatment. Prev Sci. 2013;14(2):157-68.

36. Definition of an older or elderly person. World Health Organization. http://www.who.int/ healthinfo/survey/ageingdefnolder/en. Accessed 2002.

37. Nylund KL, Asparouhov T, Muthén BO. Deciding on the number of classes in latent class analysis and growth mixture modeling: a monte carlo simulation study. Struct Equ Model. 2007;14(4):535-69.

38. Takahashi E, Moriyama K, Yamakado M. Lifestyle and glycemic control in Japanese adults receiving 
diabetes treatment: an analysis of the 2009 Japan Society of Ningen Dock database. Diabetes Res Clin Pract. 2014;104(2):e50-3.

39. Girach A, Manner D, Porta M. Diabetic microvascular complications: can patients at risk be identified? A review. Int J Clin Pract. 2006;60(11):1471-83.

40. Zoungas S, Woodward M, Li Q, et al. Impact of age, age at diagnosis and duration of diabetes on the risk of macrovascular and microvascular complications and death in type 2 diabetes. Diabetologia. 2014;57(12):2465-74.

41. Kissebah AH, Vydelingum N, Murray R, et al. Relation of body fat distribution to metabolic complications of obesity. J Clin Endocrinol Metab. 1982;54(2):254-60.

42. Wang Z, Ding L, Huang X, et al. Abdominal adiposity contributes to adverse glycemic control and albuminuria in Chinese type 2 diabetic patients: A cross-sectional study. J Diabetes. 2017;9(3):285-95.

43. $\mathrm{Xu} \mathrm{X,} \mathrm{Hall} \mathrm{J,} \mathrm{Byles} \mathrm{J,} \mathrm{Shi} \mathrm{Z.} \mathrm{Dietary} \mathrm{pattern} \mathrm{is} \mathrm{asso-}$ ciated with obesity in older people in China: data from China Health and Nutrition Survey (CHNS). Nutrients. 2015;7(9):8170-88.

44. Willi C, Bodenmann P, Ghali WA, Faris PD, Cornuz J. Active smoking and the risk of type 2 diabetes: a systematic review and meta-analysis. JAMA. 2007;298(22):2654-64.

45. Clair C, Cohen MJ, Eichler F, Selby KJ, Rigotti NA. The effect of cigarette smoking on diabetic peripheral neuropathy: a systematic review and meta-analysis. J Gen Intern Med. 2015;30(8):1193-203.

46. Xie XT, Liu Q, Wu J, Wakui M. Impact of cigarette smoking in type 2 diabetes development. Acta Pharmacol Sin. 2009;30(6):784-7.

47. Knott C, Bell S, Britton A. Alcohol consumption and the risk of type 2 diabetes: a systematic review and dose-response meta-analysis of more than 1.9 million individuals from 38 observational studies. Diabetes Care. 2015;38(9):1804-12.

48. Bishwajit G, Tang S, Yaya S, He Z, Feng Z. Lifestyle behaviors, subjective health, and quality of life among Chinese men living with type 2 diabetes. Am J Mens Health. 2017;11(2):357-64.

49. Kautzky-Willer A, Harreiter J, Pacini G. Sex and gender differences in risk, pathophysiology and complications of type 2 diabetes mellitus. Endocr Rev. 2016;37(3):278-316.

50. Lovejoy JC. The influence of sex hormones on obesity across the female life span. J Womens Health. 1998;7(10):1247-56.

51. Duin DK, Golbeck AL, Keippel AE, et al. Using gender-based analyses to understand physical inactivity among women in Yellowstone County, Montana. Eval Progr Plann. 2015;51:45-52.

52. Colberg SR, Sigal RJ, Fernhall B, et al. Exercise and type 2 diabetes: the American College of Sports Medicine and the American Diabetes Association: joint position statement. Diabetes Care. 2010;33(12):e147-67. 